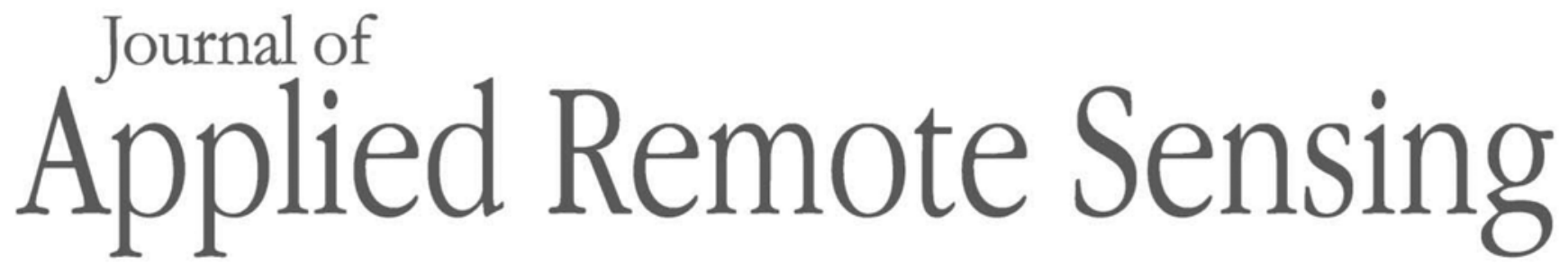

RemoteSensing.SPIEDigitalLibrary.org

\title{
Remote detection of heterogeneously spread alcohol vapors in the cabins of moving vehicles
}

Jan Kubicki

Jaroslaw Mlynczak

Krzysztof Kopczynski 


\title{
Remote detection of heterogeneously spread alcohol vapors in the cabins of moving vehicles
}

\author{
Jan Kubicki, Jaroslaw Mlynczak,* and Krzysztof Kopczynski \\ Military University of Technology, Institute of Optoelectronics, Warsaw, Poland
}

\begin{abstract}
This study concerns remote detection of alcohol vapors in cabins of moving vehicles. By means of a simplified description of propagation of two combined laser beams differently absorbed in alcohol vapors and in vehicle windowpanes, difficulties in detecting alcohol vapors in vehicle cabins using classical differential absorption method are demonstrated. Unreported in the literature, this method of remote detection of alcohol vapors in the air exhaled by a driver or a passenger in moving vehicles is proposed. The method uses two combined laser beams that illuminate different zones in the car cabin through the same windowpane. Based on the correlation between the spread of the transmission of these zones and the alcohol concentration in the air exhaled by a drunken person, it is possible to determine if the alcohol exists in the exhaled air. The effectiveness of the presented method is confirmed experimentally. (C) The Authors. Published by SPIE under a Creative Commons Attribution 4.0 Unported License. Distribution or reproduction of this work in whole or in part requires full attribution of the original publication, including its DOI. [DOI: 10.1117/1.JRS.13.044522]
\end{abstract}

Keywords: Alco-laser; alcohol detection; remote detection; alcohol detection in moving vehicles.

Paper 190707 received Sep. 10, 2019; accepted for publication Nov. 13, 2019; published online Nov. 26, 2019.

\section{Introduction}

The ability to remotely detect alcohol vapors in moving cars is particularly important to institutions responsible for traffic safety. Such detection would enable one to stop only suspected vehicles. Thanks to this, it would be possible to eliminate intoxicated drivers even on busy roads without major traffic disruption. Based on the available literature, it can be stated that work on the detection of alcohol vapors in vehicles is carried out in many countries. For a long time, Russians $^{1}$ and Poles ${ }^{2}$ have been working on it. In the United States, the DADSS research program brings together the Automotive Coalition for Traffic Safety and the National Highway Traffic Safety Administration in one of the most important government and private sector partnerships to develop a system for alcohol detection in human blood. The program is exploring two different technologies for installation in new vehicles: a breath-based system and a touch-based system. ${ }^{3}$ Also some works in India ${ }^{4}$ and China ${ }^{5}$ concerning this subject were recently published. However, there are no commercially available devices for remote detection of alcohol vapors in moving vehicles.

The idea of remote detection of alcohol vapors is a special case of remote detection of vapors and gases with the help of the monochromatic radiation absorbed in these vapors. These issues are described in Refs. 6-8, whereas remote detection of alcohol vapors in moving vehicles is described in Refs. 9-11. Several patent applications concerning this problem were also submitted. ${ }^{1,2,12}$ In Refs. 5 and 13, special attention is paid to the need to eliminate disturbances caused by the overlapping of spectrum bands of alcohol vapors with spectral bands of other vapors and gases that may occur in the cabins of tested vehicles. Also the need to increase the possibility of detecting the lowest concentrations of alcohol vapors is highlighted. In turn, in Ref. 14, the problem of car windows was clearly noticed. It was shown that windowpanes strongly suppress medium-infrared radiation used to detect alcohol vapors. In addition, the transmission of radiation in car windowpanes, especially those that are tinted, strongly depends on the wavelength.

*Address all correspondence to Jaroslaw Mlynczak, E-mail: jaroslaw.mlynczak@wat.edu.pl 
Therefore, with a large variety of windowpane thicknesses and tints, it is not clear whether the laser beam is absorbed by alcohol vapors or by windowpanes.

In this paper, a method of remote detection of alcohol vapors in moving vehicles that solves the presented problem was proposed.

\section{Physical Phenomena Used for Remote Detection of Vapors}

For better understanding of problems related to the remote detection of alcohol vapors in vehicle cabins, an analysis of the physical phenomena occurring during illuminating these cabins by laser beams was carried out. It should be noticed that there is always water vapor and carbon dioxide in the illuminated cabins. Sometimes there may be additional vapors and gases of some chemical compounds; however, in the presented analysis, they are not considered. Transmission spectra of the water vapors, carbon dioxide, alcohol vapors, and the transmission spectrum of the Pilkington untinted windowpane (thickness of $3.15 \mathrm{~mm}$ ) as well as the wavelengths of selected lasers are shown in Fig. 1. The measurements of the transmission were done using a spectrometer. The water and alcohol saturated vapors as well as carbon dioxide were put into a special cuvette and kept in temperature of $20^{\circ} \mathrm{C}$.

As the source of laser radiation, two commercially available interband cascade lasers IF3420CM1 and IF3550CM1 manufactured by Thorlabs were chosen. The lasers emitted radiation at 3.42- and 3.55- $\mu \mathrm{m}$ wavelengths, respectively, with bandwidths of $60 \mathrm{~nm}$.

Based on the presented figure, the spectra of these lasers practically do not coincide with the absorption spectra of carbon dioxide and water vapor. Also the transmission of their radiation is different in alcohol vapors, whereas it is similar in the windowpane. Therefore, it seems that using classical differential absorption of selected lasers it will be possible to detect alcohol vapors in the cabin of every vehicle regardless of the thickness of its windowpane. This supposition should, of course, be verified experimentally, but earlier it is necessary to more thoroughly follow the process of illumination the car cabin using two laser beams.

For this purpose, a simplified diagram of the discussed situation, shown in Fig. 2, was used.

When the cabin with windows is illuminated by two combined beams of light $I_{01}$ and $I_{02}$, each of them is successively suppressed by the first window, diagnosed space, and the second window. In a simplified way (by omitting reflections and assuming linear approximation of the absorption of radiation), this is shown at the bottom of Fig. 2.

In the presence of alcohol vapors, the radiation beam of wavelength $\lambda$ will be suppressed according to the Lambert-Beer law expressed in the form of the following equation:

$$
\frac{\mathrm{d} I_{\lambda}}{\mathrm{d} x}=-k_{\lambda} I_{\lambda} C(x)
$$

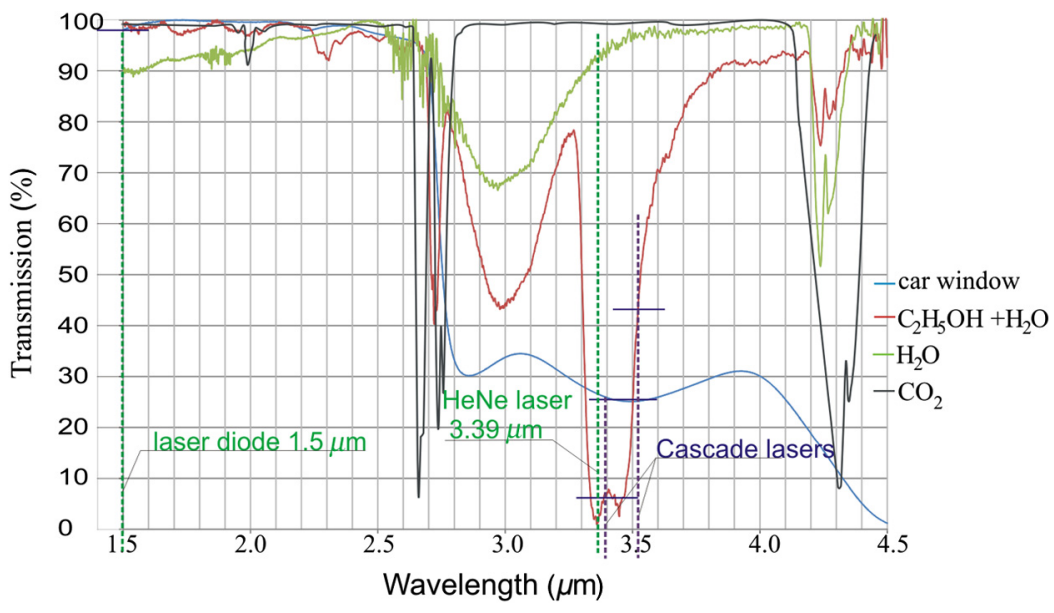

Fig. 1 Spectral characteristics of car windowpane (Pilkington), $\mathrm{CO}_{2}, \mathrm{H}_{2} \mathrm{O}$, alcohol, and lasers operating at 3.39-, 1.5-, 3.45-, and 3.59- $\mu \mathrm{m}$ wavelengths. ${ }^{15}$ 
Kubicki, Mlynczak, and Kopczynski: Remote detection of heterogeneously spread alcohol vapors...

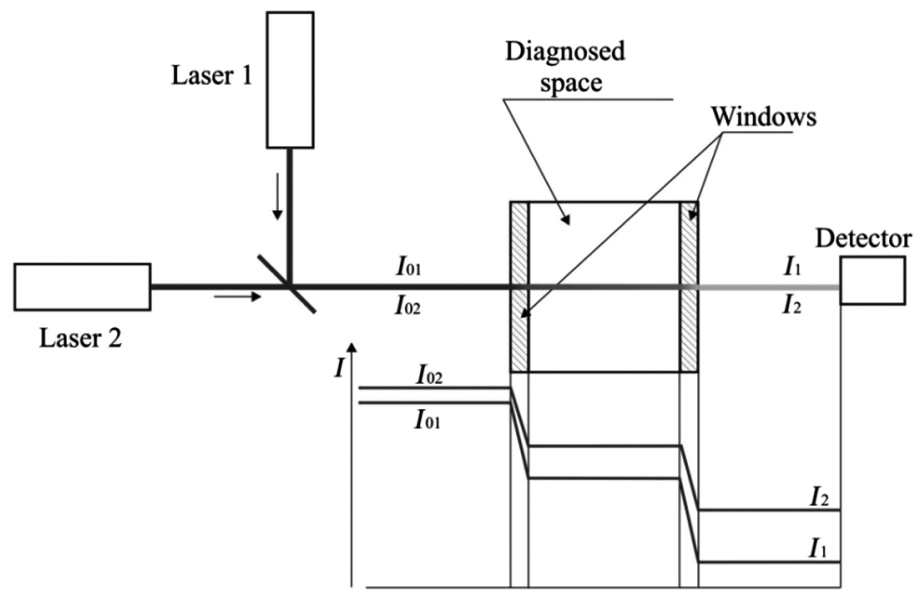

Fig. 2 Diagram of the setup for illuminating cabin with windows by two laser beams.

where $I_{\lambda}$ is the radiation intensity of wavelength $\lambda, k_{\lambda}\left(\mathrm{dm}^{3} \mathrm{~g}^{-1} \mathrm{~cm}^{-1}\right)$ is the mass absorption coefficient, and $C(x)\left(\mathrm{g} \mathrm{dm}^{-3}\right)$ is the mass concentration of alcohol vapors that depends on the distance $x$ along the direction of laser beam propagation.

By limiting the impact of the windowpanes only to their transmission depending on the wavelength $\lambda$ and integrating the Eq. (1) along the cabin interval $l$, the radiation intensity after passing through the cabin can be expressed as

$$
I_{\lambda}=I_{0 \lambda} T_{\lambda} e^{-k_{\lambda} \bar{C} l}
$$

where $T_{\lambda}$ is the transmission of the radiation of wavelength $\lambda$ for both windowpanes and

$$
\bar{C}=\frac{1}{l} \int_{0}^{l} C(x) \mathrm{d} x
$$

is the average concentration of the absorbing substance.

One can introduce the following notation.

For 3.42- $\mu \mathrm{m}$ laser beam: $I_{\lambda}=I_{1}, T_{\lambda}=T_{1}, k_{\lambda}=k_{1}$.

For 3.55- $\mu \mathrm{m}$ laser beam: $I_{\lambda}=I_{2}, T_{\lambda}=T_{2}, k_{\lambda}=k_{2}$.

Thus for the presented laser beams, Eq. (2) takes the form:

$$
\begin{aligned}
& I_{1}=I_{01} T_{1} e^{-k_{1} \bar{C} l}, \\
& I_{2}=I_{02} T_{2} e^{-k_{2} \bar{C} l},
\end{aligned}
$$

where $T_{1}, T_{2}$ are the transmissions of 3.42- and 3.55- $\mu \mathrm{m}$ radiation for both windows.

From these equations, the following equation can be derived:

$$
\frac{I_{2}}{I_{1}}=\frac{I_{02} T_{2}}{I_{01} T_{1}} e^{-\left(k_{2}-k_{1}\right) \bar{C} l}=\frac{I_{02} T_{2}}{I_{01} T_{1}} e^{\left(k_{1}-k_{2}\right) \bar{C} l} .
$$

Since only the transmission of the diagnosed space has the information concerning the alcohol concentration, a normalized value of $I_{1}$ can be introduced:

$$
I_{1}^{n}=I_{1} \cdot \frac{I_{02} T_{2}}{I_{01} T_{1}}
$$


Then Eq. (6) takes the form:

$$
\frac{I_{2}}{I_{1}^{n}}=e^{\left(k_{1}-k_{2}\right) \bar{C} l}
$$

For small values of average concentrations $\bar{C}$ (and such concentrations are interesting for the detection of alcohol vapors), this equation can be linearized by writing it in the form:

$$
\frac{I_{2}}{I_{1}^{n}} \approx 1+\delta
$$

where

$$
\delta=\left(k_{1}-k_{2}\right) \bar{C} l,
$$

thus

$$
\bar{C}=\frac{\delta}{\left(k_{1}-k_{2}\right) l}
$$

Based on Eqs. (7) and (9), for small concentrations, one can write

$$
\delta=\frac{I_{2}}{I_{1}} \frac{I_{01} T_{1}}{I_{02} T_{2}}-1
$$

When using a linear detector, the radiation intensity $I$ can be replaced with the appropriate electrical signals $S$ from this detector and the parameter $\delta$ can be determined based on the following equation:

$$
\delta=\frac{S_{2}}{S_{1}} \frac{S_{01} T_{1}}{S_{02} T_{2}}-1
$$

It can be seen that in the general case, in order to determine the parameter $\delta$, one should measure the signals from the detector corresponding to the first and second laser beam for the diagnosed space inserted in the path of the beams and for its absence, respectively. Moreover, the transmission of both laser beams through both windowpanes should be known.

\section{Experimental Verification of Possibility to Detect Alcohol Vapors Using Interband Cascade Lasers Operating at 3.42 and $3.55 \mu \mathrm{m}$}

Generally for random cars, the transmission of radiation of chosen lasers by windowpanes is not known. However, analyzing Fig. 1, one may wonder if the transmission of their radiation through the window panes of popular cars is close enough to assume $T_{2}=T_{1}$. In such situation, Eq. (13) could take the form:

$$
\delta=\frac{S_{2}}{S_{1}} \frac{S_{01}}{S_{02}}-1
$$

However, it should be noted that for a cabin without alcohol vapors, according to the Eq. (10), parameter $\delta$ should be equal to zero. To check if this is the case, an experiment was carried out according to the diagram shown in Fig. 3.

In the presented diagram, like in the case shown in Fig. 2 without alcohol vapors, the combined laser beams after going through the car pane two times fell on the detector, giving the appropriate signals proportional to the intensity of radiation.

The system uses two interband cascade lasers from Thorlabs, mentioned in Sec. 2, emitting radiation at 3.42- and 3.55- $\mu \mathrm{m}$ wavelengths with a power of $\sim 20 \mathrm{~mW}$. The lasers were powered by electrical pulses at frequencies of 50 and $80 \mathrm{kHz}$, respectively. Beams of the cascade lasers 




Fig. 3 Diagram of the system for verification of the possibility to detect alcohol vapors using interband cascade lasers operating at 3.42 and $3.55 \mu \mathrm{m} .{ }^{15}$

were combined by means of a light-dividing plate into one track into which, at the time of adjusting the system, the beam of red light from the laser diode was additionally incorporated. To detect the optical signal the HgCdTe detector from the VIGO System company was used. The electrical signals from the detector were sent into the data processing system, which in digital form passed them to a computer containing an appropriate program to determine, based on a hundred measurements, the average amplitude of the first harmonic of the detector signals.

Each measurement was carried out with the windowpane inserted in the beam path and then after a time of several seconds, without the windowpane. Based on Ref. 16, in which the described set of lasers was used and tested, it can be stated that in the aforementioned time frame of the order of several seconds, the power ratio of the laser beams could not change $>0.02 \%$.

The values of signals $S_{1}, S_{2}, S_{01}$, and $S_{02}$ were measured with accuracy of at least two orders of magnitude higher. Thus the value of $0.02 \%$ can be taken as the upper limit of measurement error for these signals, that is

$$
\frac{\Delta S_{1}}{S_{1}} \approx \frac{\Delta S_{2}}{S_{2}} \approx \frac{\Delta S_{01}}{S_{01}} \approx \frac{\Delta S_{02}}{S_{02}} \approx 0.0002
$$

By inserting the values of the measured signals into Eq. (14), one can determine the parameter $\delta$. (Its real value according to Eq. (10) should be equal to zero, whereas the nonzero value may result mainly from the adoption of identical transmissions of both beams in both windowpanes.) Based on the well-known theory of measurement errors, the accuracy of determination of the parameter $\delta$ should be

$$
\Delta \delta=\left|\frac{\partial \delta}{\partial S_{1}}\right| \Delta S_{1}+\left|\frac{\partial \delta}{\partial S_{2}}\right| \Delta S_{2}+\left|\frac{\partial \delta}{\partial S_{01}}\right| \Delta S_{01}+\left|\frac{\partial \delta}{\partial S_{02}}\right| \Delta S_{02}
$$

Making appropriate transformations of this equation and substituting numerical values, it can be assumed that $\Delta \delta<0.001$.

The results for different windowpanes of popular cars are shown in Table 1.

Based on Refs. 16 and 17, it can be stated that for alcohol vapor in a cuvette of the length of $140 \mathrm{~cm}$ (similar to the car's cabin width), for alcohol concentrations comparable to the concentration in the air exhaled by a drunk person and equal to 0.1 and $0.2 \mathrm{mg} / \mathrm{dm}^{3}$, the parameter $\delta$ should be equal to 0.052 and 0.176 , respectively. Additionally, taking into account the fact that due to inhomogeneities and air blowing, the average concentration of alcohol vapors in a car will be much smaller than in the air exhaled directly by a drunk person, one must be able to determine the actual value of the parameter $\delta$ at much lower levels. Thus it can be seen from Table 1 that for most cars it is not possible to detect alcohol vapor at such low concentrations without knowing the transmission of the laser beams by their windowpanes. The transmission of the chosen 3.42and 3.55- $\mu \mathrm{m}$ lasers for individual windowpanes is so different that it distorts the $\delta$ parameter by a value greater than its value for the average concentration of alcohol vapors one wants to detect. 
Kubicki, Mlynczak, and Kopczynski: Remote detection of heterogeneously spread alcohol vapors...

Table 1 Values of parameter $\delta$ for different windowpanes calculated by means of Eq. (14).

\begin{tabular}{lcc}
\hline \hline Car make & Thickness of the car window $(\mathrm{mm})$ & Values of $\delta$ \\
\hline Golf & 4 & 0.19 \\
Toy. Yaris & 3 & 0.10 \\
VW Passat & 4 & 0.02 \\
KIA & 3.5 & 0.11 \\
Clio & 3 & 0.19 \\
Octavia & 4 & 0.03 \\
Mondeo & 3 & 0.17 \\
Astra & 4 & 0.29 \\
Corsa & 4 & 0.30 \\
Fabia & 3.5 & 0.21 \\
Focus & 3 & 0.13 \\
Avensis & 3 & 0.04 \\
Toy. Rav4 & 3 & 0.17 \\
Fiat Duca not tinted & 3 & 0.03 \\
Fiat Duca tinted & 3 & 0.11 \\
VW Polo & 4 & 0.23 \\
\hline \hline
\end{tabular}

In addition, based on the analysis of physical phenomena occurring during the emission of radiation in interband cascade lasers (especially considering the relatively broad spectrum of emitted radiation), it seems that it will also be impossible to detect alcohol vapors in random cars with the use of tunable lasers.

\section{Concept of the Remote Detection of Heterogeneously Spread Alcohol Vapors}

In known publications concerning the detection of alcohol vapors in vehicle cabins, the average value of concentration of these vapors in the entire cabin was assumed, without considering its spatial distribution. It is known, however, that alcohol vapors are exhaled by the drunk driver in a particular place. At the same time, air flows into the cabin without alcohol vapors from a specific place (including the openings of the ventilation system). Therefore, the concentrations of alcohol vapors in different parts of the cabin must be different. Such situation was shown in Fig. 4.

During the passage of the car through the control system, two combined laser beams differently absorbed by the alcohol vapors, through the same set of front side windowpanes, illuminate subsequent zones of the car cabin. As a result, when scanning various areas of the car cabin through homogeneous side windowpanes, one should obtain different values of $\delta$ for subsequent illuminated zones, related only to heterogeneously distributed vapors of alcohol. Thus according to Eq. (14), for zone $n$, the value of $\delta$ should be equal to

$$
\delta_{n}=\frac{S_{2 n}}{S_{1 n}} \frac{S_{01}}{S_{02}}-1,
$$

where $S_{2 n}, S_{1 n}$ are the signals $S_{2}$ and $S_{1}$ for zone $n$.

Thus on the basis of the heterogeneity of the parameter $\delta$ in the illuminated zones, one can conclude about the possibility of appearance of alcohol vapor in the car cabin. 


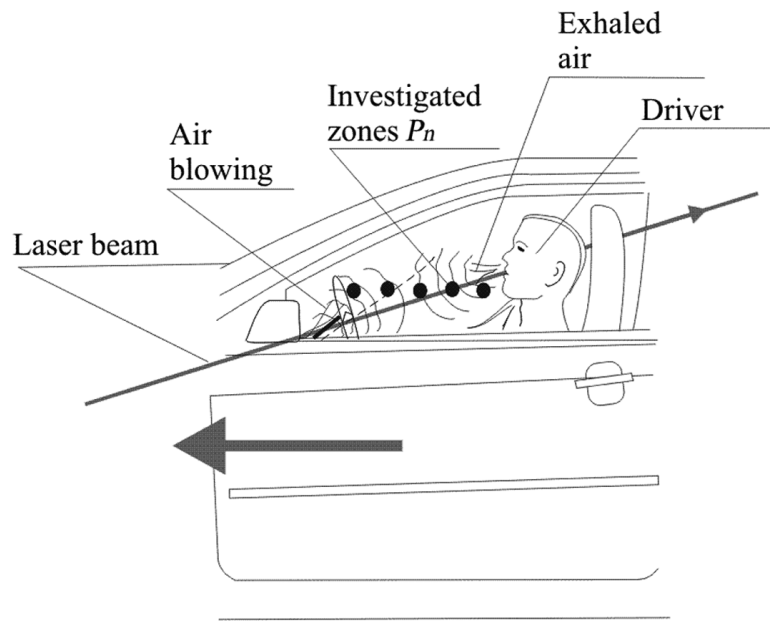

Fig. 4 Schematic drawing of the investigated car cabins.

Analyzing Eq. (15) more closely, one can see that in order to determine $\delta$, it is necessary to measure the $S_{01}$ and $S_{02}$ signals before the car enters the scanning area and to assume that the laser work is stable enough. However, taking into account the fact that we are only interested in changes in the $\delta$ value and not its absolute value, it is convenient to normalize the signals by referring them to their mean values from all the illuminated $P_{n}$ zones for a specific pair of side windowpanes, instead of normalizing the $S_{1}$ signal with respect to the $S_{01}$ signal and $S_{2}$ signal with respect to the $S_{02}$ signal. Thanks to this, getting rid of the presented problem connected with reading and analyzing relatively high signals $S_{01}$ and $S_{02}$ with respect to the $S_{1}$ and $S_{2}$ signals, Eq. (15), for a given $P_{n}$ zone can be replaced by the following expression:

$$
\delta_{n}^{r}=\frac{S_{2 n}}{S_{1 n}} \frac{\sum_{n} S_{1 n}}{\sum_{n} S_{2 n}}-1
$$

where $\delta_{n}^{r}$ is the relative parameter $\delta$ for zone $P_{n}, S_{1 n}$ is the detector signal corresponding to the radiation more strongly absorbed by the alcohol vapors in the zone $P_{n}, S_{2 n}$ is the detector signal corresponding to the radiation less absorbed by the alcohol vapors in the zone $P_{n}$, and $\Sigma n$ is the adding up the appropriate signals from all zones.

However, in special conditions when there is moisture or dust on the windowpanes characterized by absorption that depends on the wavelength of the radiation or when there are electrical disturbances in the area, there may be some spreads of $\delta$ not associated with the alcohol vapors in the cabin of the examined vehicle.

This problem can be solved by raising the threshold value of the spread of $\delta$ by the amount proportional to the average value of the spread for a given number of cars checked successively before the current car. Of course, this will be at the expense of the ability to detect the minimum average concentrations of alcohol vapors.

\section{Experimental Confirmation of the Possibility to Detect Heterogeneously Spread Alcohol Vapors}

The presented concept of detecting alcohol vapors in the cabins of moving vehicles was verified in an experimental setup shown in Fig. 5.

The system consisted of a stationary transmitter, described in Sec. 3, emitting two combined laser beams differently absorbed by alcohol vapor. The beams illuminated subsequent areas $P_{n}$ of the cabin of the passing car and fell on the stationary receiver placed on the opposite side of the car, also described in Sec. 3. After passing through a diaphragm with a diameter of $10 \mathrm{~mm}$, the beams were focused by a spherical mirror with a $20-\mathrm{cm}$ focal length on the $\mathrm{HgCdTe}$ detector characterized by $2 \times 2 \mathrm{~mm}$ active area. 


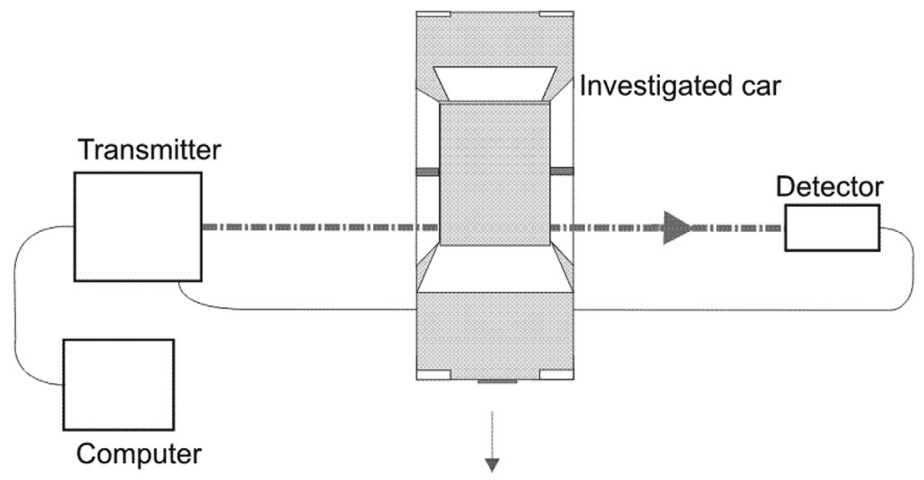

Fig. 5 Experimental setup.

Based on the read signals, the computer, using appropriate program incorporating Eq. (16), calculated the values of parameter $\delta_{n}^{r}$ for $50 P_{n}$ zones illuminated by the vehicle's side windowpanes. Then for the obtained sequence of values of $\delta_{n}^{r}$, its standard deviation $\sigma$ was determined.

In the tests, the Ford Fiesta and Renault Captur were used. A drunk person simulator was installed on the seat next to the driver, who, at 20 times/ min, blew $0.5 \mathrm{~L}$ of air at the level of the head toward the floor with alcohol vapors of known (measured previously) concentration. Due to the relatively slow signal registration and processing system used in the experiment, cars had to move at low speeds below $15 \mathrm{~km} / \mathrm{h}$. The measurements for a given concentration of alcohol vapors were carried out three times for each car. For each measurement, the standard deviation $\sigma$ was determined. For each concentration of alcohol and each car, the averaged standard deviation $\bar{\sigma}$ (from three measurements) was calculated. The results of the experiments are presented in Table 2 and in Fig. 6.

Table 2 Results of measurements and calculations.

\begin{tabular}{|c|c|c|c|c|}
\hline \multirow[b]{2}{*}{$\begin{array}{l}\text { Concentration of alcohol } \\
\text { vapors }\left(\mathrm{mg} / \mathrm{dm}^{3}\right)\end{array}$} & \multicolumn{2}{|c|}{ Ford Fiesta } & \multicolumn{2}{|c|}{ Renault Captur } \\
\hline & $\begin{array}{l}\text { Standard } \\
\text { deviation } \sigma\end{array}$ & $\begin{array}{l}\text { Averaged standard } \\
\text { deviation } \bar{\sigma}\end{array}$ & $\begin{array}{l}\text { Standard } \\
\text { deviation } \sigma\end{array}$ & $\begin{array}{c}\text { Averaged standard } \\
\text { deviation }\end{array}$ \\
\hline \multirow[t]{3}{*}{0.0} & 0.034 & 0.033 & 0.042 & 0.038 \\
\hline & 0.035 & & 0.033 & \\
\hline & 0.030 & & 0.038 & \\
\hline \multirow[t]{3}{*}{0.3} & 0.043 & 0.041 & 0.042 & 0.042 \\
\hline & 0.038 & & 0.043 & \\
\hline & 0.042 & & 0.041 & \\
\hline \multirow[t]{3}{*}{0.4} & 0.040 & 0.040 & 0.043 & 0.044 \\
\hline & 0.041 & & 0.046 & \\
\hline & 0.039 & & 0.043 & \\
\hline \multirow[t]{3}{*}{1.1} & 0.037 & 0.045 & 0.047 & 0.048 \\
\hline & 0.050 & & 0.047 & \\
\hline & 0.049 & & 0.051 & \\
\hline \multirow[t]{3}{*}{2.3} & 0.052 & 0.048 & 0.046 & 0.050 \\
\hline & 0.045 & & 0.045 & \\
\hline & 0.047 & & 0.058 & \\
\hline
\end{tabular}




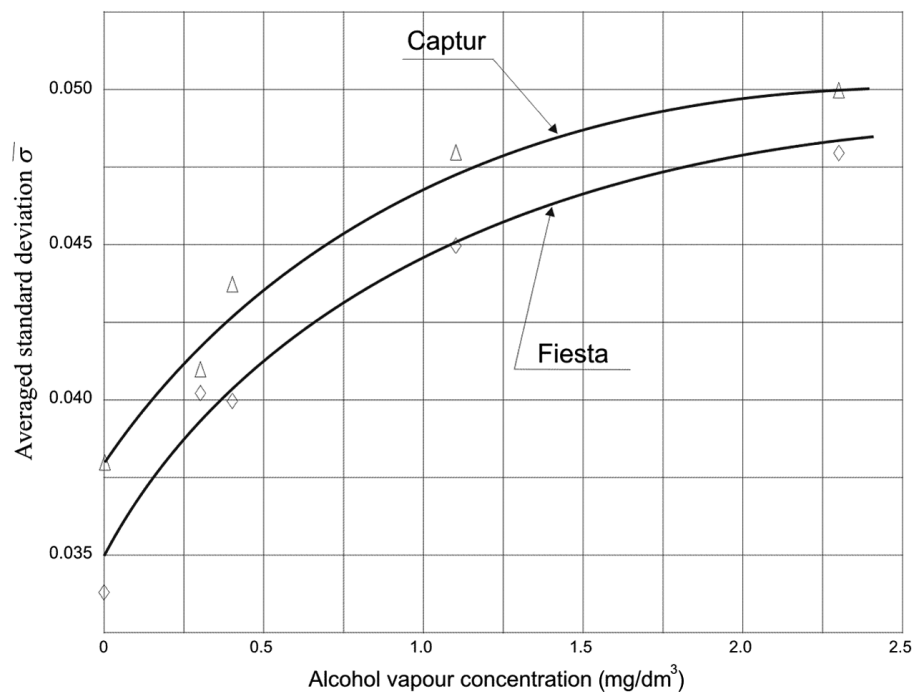

Fig. 6 Graphs of averaged standard deviations of $\bar{\sigma}$ for investigated cars as a function of the concentration of alcohol vapors blown out from the simulator.

For the screened cars, a clear monotone dependence of the averaged standard deviation $\bar{\sigma}$ (for sequences of $\delta_{n}^{r}$ ) on the concentration of alcohol vapors in the air blown out by the simulator can be seen.

Figure 6 shows that the values of the parameter $\bar{\sigma}$, for both cars, differ $<20 \%$ from the values of the approximating line. Thus assuming that $\frac{\Delta \bar{\sigma}}{\bar{\sigma}}$, the alcohol concentration in cars $C$ and the limits of its possible values in function of $\bar{\sigma}$ parameter can be calculated. The results of the calculation are presented in Fig. 7.

The presented graphs show that for a specific car, after determining $\bar{\sigma}$ parameter, the concentration of alcohol vapors in exhaled air as well as its uncertainty can be determined.

\section{Comments on the Possibilities of Building a Professional Device}

The experiment presented in this paper shows that there is a real chance to build the device based on the proposed method for detecting alcohol vapors in moving cars. There are of course many problems to solve. The graphs in Figs. 6 and 7 show that in each car we can deal with a different distribution of concentration of alcohol vapors, and therefore the corresponding graphs are shifted relative to each other (Fig. 6). The results can be averaged but this will introduce larger errors and thus increase the detection threshold. Therefore, it seems that using a camera that will recognize the brand of the car is a better solution. Assigning the parameter $\bar{\sigma}$ to this brand, the device will more reliably classify the car as "suspicious" or not.

In the proposed device, it is necessary to solve many other problems such as the accuracy of coverage of beam paths and their spatial distribution, the speed of the electronic system and its
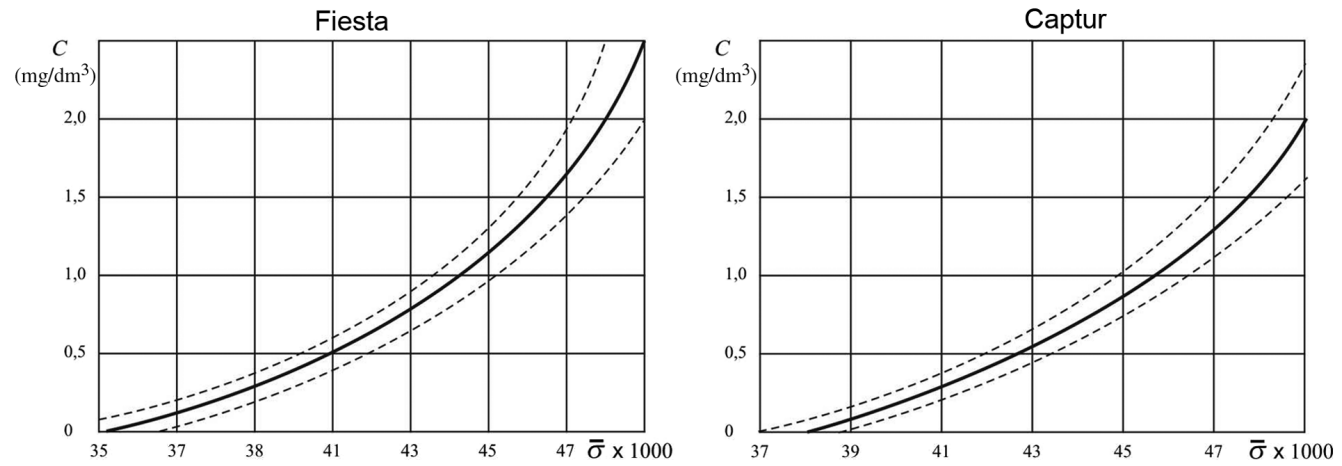

Fig. 7 Graphs of the alcohol concentration in cars $C$ (solid lines) and the limits of its possible values (dashed lines) in function of parameter. 
immunity to interferences, synchronization of the start and end of the diagnostic of the car cabin, and elimination of measurements from zones where there is partial shading of the laser beams. The size of the car or the position over the ground at which the windowpane is mounted is a separate issue. However, it can be imagined that the position of the laser beam over the ground is changed by a special elevation optical system. However, these are purely technical problems, which should be easily solved in a final device.

There are, of course, many questions about the adverse impact of various factors on the ability to detect alcohol vapors applying the described method, such as the effect of changing the angle of the car relative to the direction of the laser beams or the impact of the pressure gradient outside the car at its higher speeds. It should be noted, therefore, that the difference between the wavelengths of the laser beams is small, so neither the inclination angle of the windowpanes nor the pressure gradients at higher speeds should cause separation of these beams. Thus the mentioned factors can cause the same changes of the intensity of both laser beams and, as shown in Eq. (12), they cannot distort the parameter $\delta$ and the parameter $\delta_{n}^{r}$.

The issue of eye safety is also extremely important. The power of the applied lasers exceeds $20 \mathrm{~mW}$, but the optical systems to combine and to collimate the beams, as well as diaphragms reduce the total power to below $5 \mathrm{~mW}$. The estimated and measured power density at $2 \mathrm{~m}$ from the transmitter is below $50 \mathrm{~W} / \mathrm{m}^{2}$. Meanwhile, according to Laser Safety Standard EN 60825, the maximum permissible exposure at the cornea of eye at this wavelength is $1000 \mathrm{~W} / \mathrm{m}^{2}$. Thus even with the car window open and looking directly at the laser beam, there is no danger to eyes.

When assessing the suitability of the device, it seems that it will not only determine the driver's state of intoxication but will also not determine the average alcohol vapor concentration in the cabin. However, as was already mentioned in Sec. 1, the objective of the device is not to determine the average concentration of alcohol vapors in the vehicle (which may also come from various sources not related to the driver). The purpose of using the device is only to reduce the population of suspected cars intended to be stopped for direct control of the driver, which will enable to check vehicles even on a busy road without major traffic disruptions.

When assessing the suitability of the device, there may be allegations that the device will not only determine the driver's intoxication level, but will also not accurately determine the average concentration of alcohol vapor in the cabin. As was already mentioned in Sec. 1, the aim of the device is not to determine the average concentration of alcohol vapors in the cabin (which may also come from various sources not related to driver intoxication). The aim of using the device is only to reduce the population of suspicious cars that are intended to be stopped for the purpose of direct control of the driver, which will enable to control vehicles even on a busy road without major traffic disturbance.

Of course, it is not possible to develop the system that is universal for every situation. High humidity in the cabin, despite the slight coverage of the water and alcohol vapors spectra shown in Fig. 1, should not significantly interfere with the detection of alcohol vapors because the water vapors are homogeneously distributed in contrast to alcohol vapors. However drops of water, snow, or dust on the windowpanes and in the air may limit usability of the device. It should also be noted that there are many accidental situations during the measurements. There may be a different kind of ventilation as well as driver's silhouette in each car. Therefore, only on the basis of studies of a sufficiently large car population, it will be possible to put error bars and uncertainty of measurements on the graph shown in Fig. 7. Experiments with large car population in real situations (on a real road) would enable to determine detection limits and false positive rate.

\section{Conclusion}

In this work, based on the experiments using large variety of front side windows and based on the described analysis, the effectiveness of alcohol detection in car cabins applying classic methods of differential absorption was questioned. At the same time, applying the method based on the heterogeneous distribution of concentration of alcohol vapors in car cabins, not only alcohol vapors were detected but also their concentrations in the air exhaled by the driver or passenger were determined with appropriate accuracy. Thus applying the presented method, there is a real chance to build a universal device for remote detection of alcohol vapors in moving vehicles. 


\section{References}

1. O. Ershov, A. Nadezdinskii, and A. Berezin, "Remote gas molecule detector," U.S. Patent No. 0,160,173 A1 (2003).

2. P. Kluczynski and S. Lundqvist, "Method and apparatus for remote detection of alcohol vapours in the atmosphere," U.S. Patent No. 9,068,885 B2 (2015).

3. A. K. Zaouk et al., "Driver alcohol detection system for safety (DADSS)," Paper Number 15-0276, https://www-esv.nhtsa.dot.gov/Proceedings/24/files/Session\%2012\%20Oral.pdf (20 November 2019).

4. A. Gupta et al., "Alcohol detection with vehicle controlling," Int. J. Eng. Manage. Res. 6, 20-23 (2016).

5. H. Gao et al., "Stand-off detection of ethanol vapor based on a tunable ICL," Opt. Express 26, 21433-21442 (2018).

6. R. M. Silverstein, G. C. Bassler, and T. C. Morrill, Spectrometric Identification of Organic Compounds; Wiley, New York (1991).

7. W. Demtröder, Laser Spectroscopy, Springer-Verlag, Berlin (2002).

8. A. Beil, R. Daum, and T. Johnson, "Detection of chemical agents in the atmosphere by passive IR remote sensing in internal standardization and calibration architectures for chemical sensors," Proc. SPIE 3856, 44-56 (2000).

9. T. A. Alobadi and D. W. Hill, "A helium-neon laser infrared analyser for alcohol vapour in the breath," J. Phys. E Sci. Instrum. 8, 30-32 (1975).

10. M. Azzazy et al., "Remote sensor to detect alcohol impaired drivers," in IEEE Lasers and Electro-Opt. Soc. Annu. Meeting, 8th Annu. Meeting Conf. Proc., Vol. 2, pp. 320-321 (1995).

11. A. Nadezhdinskii et al., "Application of near-IR diode lasers for measurement of ethanol vapor," Spectrochim. Acta A 55, 2049-2055 (1999).

12. S. Nishida, "Alcohol detector in vehicle," Patent no. JP2000230900 (A) (2000).

13. H. Geng et al., "Ethanol vapor measurement based on tunable diode laser absorption spectroscopy," Acta Phys. Sin. 63, 043301 (2014).

14. J. Kubicki, J. Mlynczak, and K. Kopczynski, "Application of modified difference absorption method to stand-off detection of alcohol in simulated car cabins," J. Appl. Remote Sens. 7(1), 073529 (2013).

15. J. Mlynczak et al., "Assessment of the application of cascade lasers to stand-off detection of alcohol vapors in moving cars," J. Appl. Remote Sens. 10, 046010 (2016).

16. J. Mlynczak, J. Kubicki, and K. Kopczynski, "Stand-off detection of alcohol vapors exhaled by humans," Sensors 18, 1310 (2018).

17. K. Kopczynski et al., "Stand-off detection of alcohol vapours in moving cars," Proc. SPIE 10159, 101590Z (2016).

Jan Kubicki is a graduate from the Military University of Technology, Warsaw, Poland. In 1980, he received his $\mathrm{PhD}$ in the field of molecular lasers. Currently, he works at the Institute of Optoelectronics of Military University of Technology. He is an author and co-author of many scientific papers in the field of laser physics, laser spectroscopy, and high-power laser systems.

Jaroslaw Mlynczak received his MSc degree in 2002 and PhD in 2008 from the Military University of Technology, Warsaw, Poland, where he currently works as a scientist. His research centers on investigation of new active media and new nonlinear absorbers for UV, VIS, IR, and "eye-safe" microchip lasers as well as the development of microchip lasers for stand-off detection systems. He also participates in research concerning detection of biological and chemical agents in the environment. He is an author and co-author of many scientific and conference papers.

Krzysztof Kopczynski received his MSc degree in solid-state physics and quantum electronics and his PhD in the field of laser physics from the Military University of Technology. He is a director of the Institute of Optoelectronics at the Military University of Technology. He is a specialist in the field of solid-state lasers, microchip lasers, and laser devices for stand-off detection. $\mathrm{He}$ is an author and co-author of a few tens of scientific and conference papers. 